Journal of Clinical Investigation

Vol. 41, No. 2, 1962

\title{
STUDIES ON THE PATHOGENESIS OF MENINGITIS. II. DEVELOPMENT OF MENINGITIS DURING PNEUMOCOCCAL BACTEREMIA *
}

\author{
By ROBERT G. PETERSDORF, DAVID R. SWARNER AND MANUEL GARCIA \\ (From the Division of Allergy and Infectious Disease, Department of Medicine, Johns Hopkins \\ School of Medicine Baltimore, and Department of Medicine, University of \\ Washington School of Medicine, Seattle, Wash.)
}

(Submitted for publication August 9, 1961 ; accepted October 19, 1961)

In a previous publication we described an experimental form of pneumococcal meningitis in dogs, which was produced by introduction of type III pneumococci into the subarachnoid space through a small midline burr hole placed equidistant between the coronal and occipital sutures (1). The infection produced in this manner involved the entire neuraxis and, with the exception of marked changes in the spinal cord, closely resembled that found in man. It seems unlikely, however, that entry from the external environment is the only means by which bacteria enter the subarachnoid space. An alternative possibility is that the meninges are infected by way of the blood stream. The present paper is concerned with the development of meningitis during pneumococcal bacteremia in dogs.

With the exception of the early report by Bull (2) that intravenous administration of pneumococci to dogs was followed by meningitis, in most studies intravenous injection of a variety of microorganisms into rabbits, dogs, monkeys, and cats has not resulted in infection of the meninges (3-7). Weed, Wegeforth, Ayer and Felton (6, $8)$, however, regularly observed meningitis when cats were subjected to lumbar puncture during bacteremia with Aerobacter aerogenes, an encapsulated, gram-negative bacillus. Control animals given twice as many organisms but not subjected to lumbar puncture remained well. Infection developed only when cerebrospinal fluid (CSF) was removed 30 minutes before or after the injection of bacteria, and meningitis did not develop when lumbar puncture was performed outside this 30-minute interval. Infected animals generally became sick within 24 hours and died 24 to 48 hours later. A. aerogenes was used in

* Supported by a grant from the U. S. Public Health Service and grants from Parke, Davis \& Co. and the Upjohn Co. the majority of experiments and cats were the most commonly employed animals. However, cats could also be successfully infected with Pseudomonas aeruginosa and Salmonella paratyphosa $B$, while in rabbits infection could be produced with streptococci and meningococci. The authors postulated two requisites for development of infection: 1) the organisms had to be virulent for the meninges, and 2) a sufficiently large number of bacteria had to be inoculated.

The most obvious explanation for these results is that trauma to small meningeal blood vessels at the time of lumbar or cisternal puncture provided access for bacteria, but careful morphological studies failed to substantiate this. Furthermore, injection of hypertonic sodium chloride, compression of the jugular veins, and transient cardiac standstill during bacteremia were also followed by meningeal infection, indicating that direct injury to the meninges was not a requirement for the development of meningitis. Weed and co-workers hypothesized that occurrence of infection was facilitated by the transient fall in $\mathrm{CSF}$ pressure associated with these procedures $(6,8)$. However, accurate measurements of CSF pressure under these conditions were not made. Furthermore, the development of meningitis during jugular compression, a maneuver known to increase CSF pressure, is inconsistent with this hypothesis.

The studies to be reported deal with some of the factors influencing the development of meningeal infection during pneumococcal bacteremia with emphasis on alterations in CSF pressure.

\section{MATERIALS AND METHODS}

Animals. All experiments were performed in mongrel dogs weighing between 10 and $20 \mathrm{~kg}$. Animals were housed in individual cages and were permitted food and water ad libitum. 
Microorganism. All experiments were performed with the encapsulated strain of Diplococcus pneumoniae previously used to produce infection by the intrathecal route (1). The steps for preparing the organism for infection have been detailed previously (1).

Experimental infections. Animals were anesthetized with pentobarbital, the scalp was carefully shaved and prepared with soap, saline, and tincture of merthiolate. With the animal on its side, $4.0 \mathrm{ml}$ of an 18-hour broth culture containing between $10^{5}$ and $10^{8}$ viable pneumococci was injected into the saphenous vein. Exactly 2 minutes later, cisternal puncture was performed and $2.0 \mathrm{ml}$ of CSF was removed under aseptic conditions. This fluid was invariably sterile and contained no cells. All animals with traumatic cisternal punctures were discarded. Initially, control animals were given 2 to 4 times the inoculum of the experimental group and cisternal punctures were not performed until 24 hours later. No animal not subjected to cisternal puncture within $30 \mathrm{~min}$ utes after injection of pneumococci developed infection.

For comparison, meningitis was produced in a group of dogs by direct inoculation of organisms into the subarachnoid space through a small midline burr hole placed equidistant between the coronal and occipital sutures. This method has been detailed in a previous publication (1).

After administration of bacteria, animals were returned to their cages and cisternal punctures were performed at 24-hour intervals until death or recovery. Animals with three consecutive negative CSF cultures were considered free of infection.

Pathology. The methods for embedding and sectioning the brains, and staining the sections have been described (1).

Bacterial counts. The number of bacteria in the inocula, CSF, brain, and meninges was determined by colony counts of serial tenfold dilutions. To determine the number of bacteria in brain and meninges, autopsies were performed on the animals under aseptic conditions, the brain and its covering removed, and the meninges stripped from the underlying cortex. Each tissue was weighed on a quantitative balance, diluted to constant volume in 0.85 per cent $\mathrm{NaCl}$ and ground in a Ten Broeck grinder. Pour plates were made of serial tenfold dilutions of ground tissues.

Blood cultures. Blood was obtained from the femoral vein under aseptic conditions and cultured in trypticase soy and thioglycollate broth. Blood cultures were always taken from the limb which had not been used for injection of bacteria. For quantitative determination of bacteria $1.0 \mathrm{ml}$ of blood was added indirectly to $9.0 \mathrm{ml}$ of trypticase soy broth and pour plates of serial tenfold dilutions were made. Leukocyte counts and CSF glucose were performed as described previously (1).

CSF pressures were determined with a direct-reading spinal manometer. The manometer was left in place for 2 minutes to permit equilibration to take place. All pressures were obtained in anesthetized animals.

A bacterial vaccine made from Salmonella typhosa

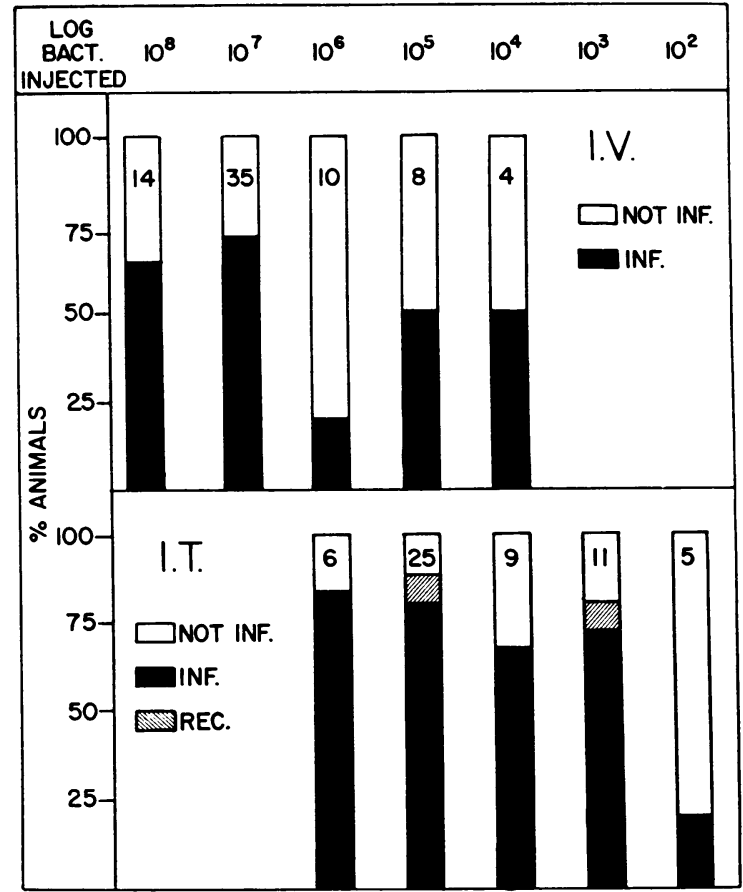

Fig. 1. Comparison of Rate of infection between DOGS INFECTED BY THE INTRAVENOUS AND INTRATHECAL Routes. None of 9 animals given $10^{3}$ and $10^{2}$ bacteria i.v. developed infection.

(strain Ty-2, Felix) ${ }^{1}$ was used in some experiments. Prior to inactivation by phenol and heat, this material contained $10^{10}$ viable bacteria. Sterile 6 per cent dextran and 50 per cent dextrose were purchased from commercial sources.

\section{RESULTS}

Incidence of infection during bacteremia. Cisternal puncture was performed on 71 animals within 2 minutes after intravenous administration of pneumococci; 42 of these animals developed meningitis (Figure 1). With the exception of the 10 animals given $10^{6}$ organisms, the incidence of infection ranged between 50 and 75 per cent, and $10^{4}$ bacteria appeared to be the minimum infecting dose. In experiments not shown in Figure 1 , five dogs were injected with $10^{3}$ and four with $10^{2}$ organisms and none of these nine dogs developed infection. All infected animals died and no spontaneous recoveries occurred. Normal control dogs were given $10^{9}$ bacteria intravenously and not subjected to cisternal puncture until 24 hours later; none developed meningitis.

1 Obtained from Dr. Maurice Landy, U. S. Public Health Service, Bethesda, Md. 
TABLE I

Relationship of number of bacteria in blood stream at the time of cisternal puncture to development of infection

\begin{tabular}{cccc}
\hline \hline & \multicolumn{2}{c}{ Per cent of dogs infected } \\
\cline { 2 - 3 } No. bacteria $/ \mathrm{ml}$ blood & $1 \mathrm{~min} *$ & 5 min* \\
\hline & $10^{3}$ & 59 & 81 \\
$10^{3}$ & 33 & 17 \\
\hline
\end{tabular}

* Time after cisternal puncture.

When pneumococci were administered intrathecally, the rate of infection was almost twice that after intravenous injection, and only 1 to 10 per cent as many bacteria was required to produce meningitis. This is not surprising in view of the dilution of the bacterial inoculum in the blood stream which would result in relatively few organisms actually reaching the subarachnoid space.

Relationship of number of microorganisms in blood stream to development of infection. While there was some relationship between the magnitude of the bacterial inoculum and the development of infection, it seemed probable that considerable variation existed in the rate at which bacteria were cleared from the blood stream in individual animals. Therefore, the number of organisms actually in the circulation at the time

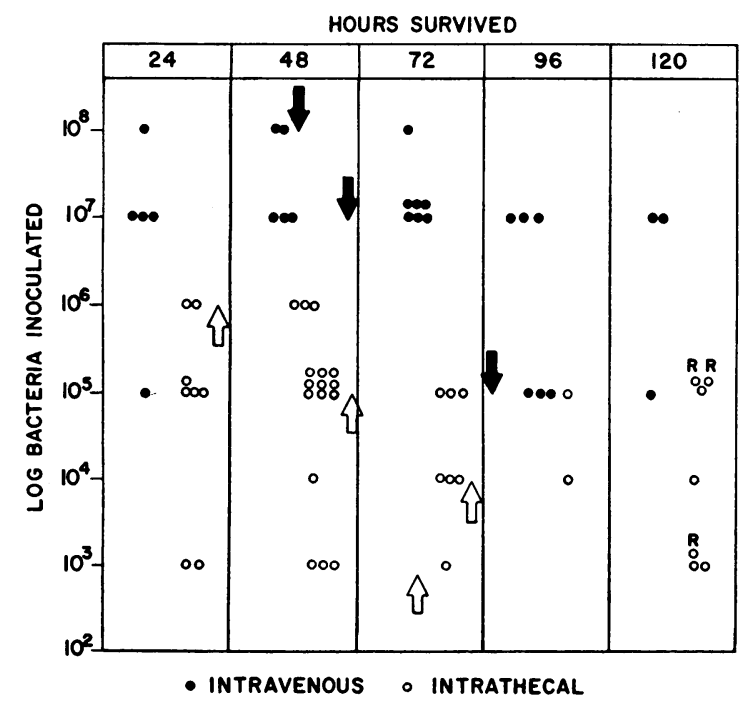

Fig. 2. Relationship OF Bacterial inOculum to LENGTH OF SURvival. There was a direct relationship between the number of organisms injected and length of survival. Arrow $=$ mean, $\mathrm{R}=$ recovered. of cisternal puncture seemed a more adequate index of the number of bacteria necessary for the development of infection.

Femoral vein punctures were performed in 32 dogs, 1 and 5 minutes after cisternal puncture ( 3 and 8 minutes after intravenous injection of bacteria) and the number of organisms per $\mathrm{ml}$ of whole blood determined by quantitative plate counts. The results are summarized in Table I and clearly demonstrate a direct relationship between the number of organisms in the blood stream and the development of meningeal infection. For example, 59 per cent of animals with more than $10^{3}$

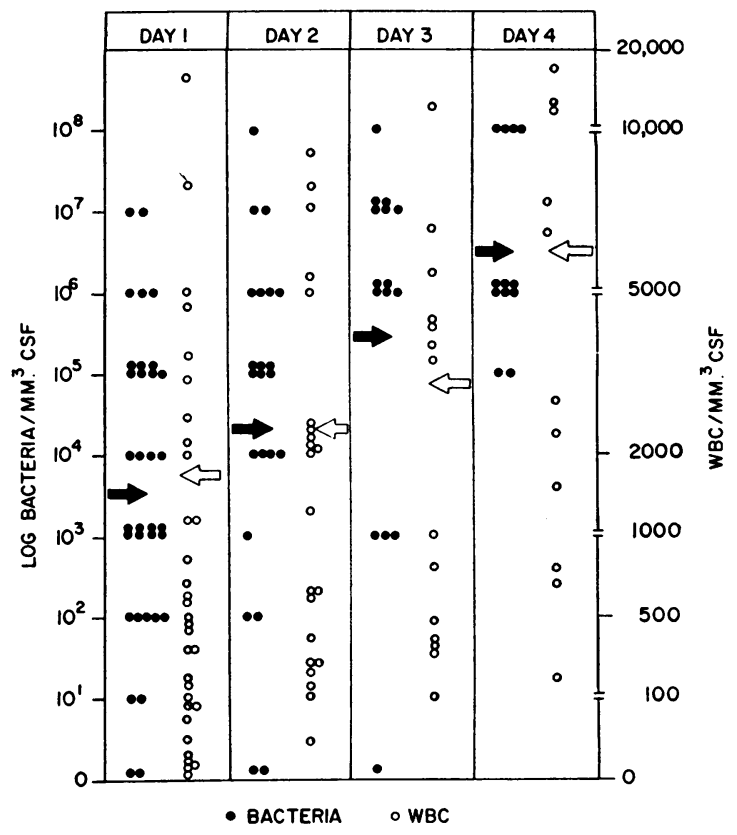

Fig. 3. Animals Developing Meningitis After inTRAVENOUS INOCULATION OF PNEUMOCOCCI. Note the persistent increase in bacteria and cells in CSF as infection progressed.

bacteria per $\mathrm{ml}$ of blood became infected within 1 minute after cisternal puncture. The rate of infection among dogs with fewer than $10^{3}$ organisms was only 33 per cent. This difference was much more striking 5 minutes after cisternal puncture; 81 per cent of animals with more than $10^{3}$ bacteria in the blood stream at that time developed infection, while only 17 per cent of those with fewer than $10^{3}$ organisms contracted meningitis.

Relationship of duration of illness to size of bacterial inoculum. There was a direct relation- 
ship between the number of organisms used to produce infection and the number of hours the animals survived. This is illustrated in Figure 2. For example, the mean survival time of dogs inoculated with $10^{8}$ bacteria was 48 hours and that of animals given $10^{7}$ pneumococci, 60 hours. Dogs injected with $10^{5}$ bacteria lived for 85 hours. When the infection was produced by direct inoculation of bacteria into the subarachnoid space, a much smaller number of organisms was required to produce the same lethal effect. Animals with a mean survival time of 48 hours had been infected with only $10^{5}$ organisms, approximately 1 per cent of the dose required to produce death by the intravenous route. Approximately the same relationship was true at other multiples.

Course of infection (Figure 3). Daily determinations of bacteria and cells in the CSF are tabulated in Figure 3. There was a progressive increase in bacteria from a mean of $5 \times 10^{3}$ per $\mathrm{ml} \mathrm{CSF}$ on the day after infection to $4 \times 10^{6}$ per $\mathrm{ml} \mathrm{CSF} 3$ days later. During the same period, leukocytes in the CSF increased from 1,800 to over 6,000 per $\mathrm{mm}^{3} \mathrm{CSF}$. The course of the intravenously induced infection is compared with that produced by the intrathecal route in Figure 4. The former showed a steady increment in bacteria and cells, while the infections produced by the intrathecal route were characterized by less consistent increases in the number of bacteria, and a heavy outpouring of leukocytes at the onset, with little further exudation as infection progressed. CSF glucose was measured in most animals and showed the expected fall.

Persistence of positive blood cultures (Figure 5). Of the dogs with meningitis, 93 per cent had pneumococci isolated from the blood at some time during the course of infection. Of the 32 animals, 75 per cent had bacteremia on the first day after inoculation of bacteria, 55 per cent on the second day, 66 per cent on the third day, and 72 per cent on the fourth day. In all instances the incidence of bacteremia was higher after intravenous introduction of pneumococci than after intrathecal infection. Also noteworthy is the finding that 4 of 26 animals that did not develop meningitis had persistent bacteremia for 24 to 48 hours after administration of pneumococci. In contrast, no animal given the organism intrathecally manifested bacteremia unless meningitis developed.

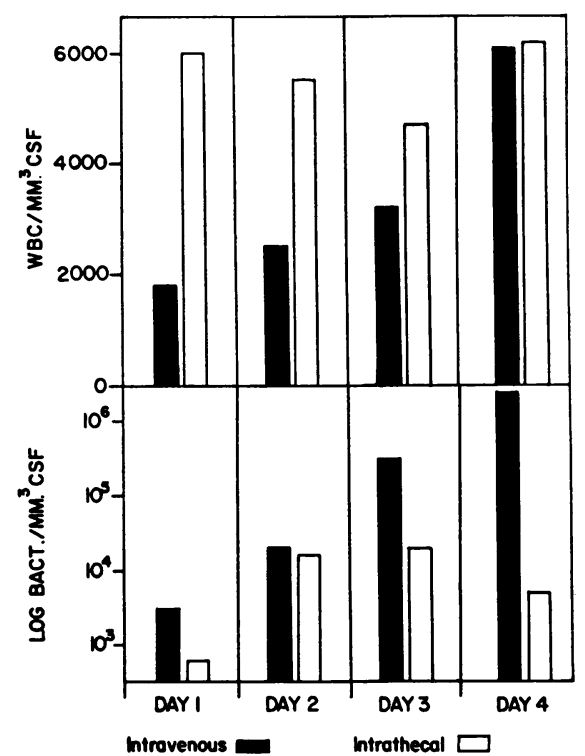

Fig. 4. Comparison of cells and bacteria in CSF AFTER INTRAVENOUS AND INTRATHECAL INFECTION.

The higher incidence of blood stream invasion and sporadic persistence of bacteremia in the animals infected intravenously are entirely consistent with the difference in route of administration. Animals were carefully examined for evidence of bacterial endocarditis and none was found.

Spread of infection within the central nervous system. It seemed of interest to determine whether the infection spread from the leptomeninges to the brain, or whether these animals de-

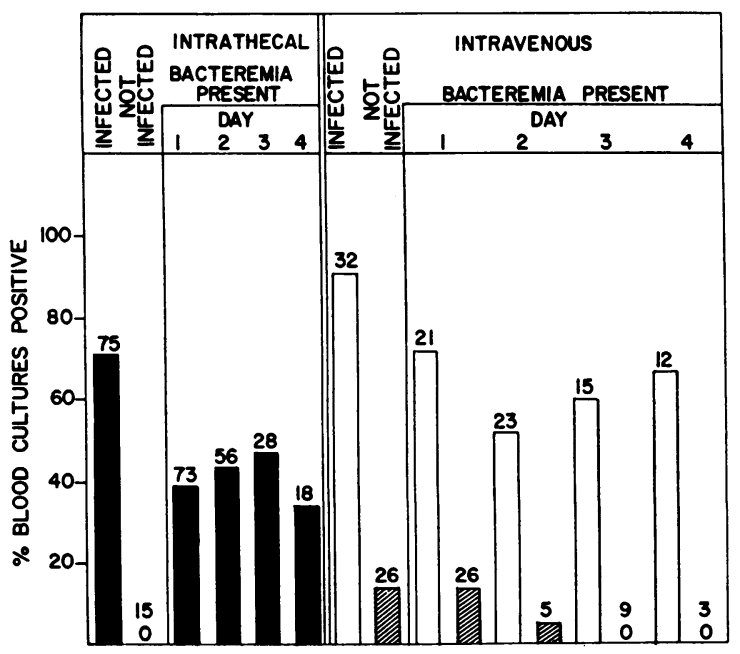

Fig. 5. INCIDENCE OF BACTEREMIA DURING PNEUMOCOCCAL MENINGitis. Note persistent bacteremia in some animals given pneumococci i.v. that failed to develop infection. 


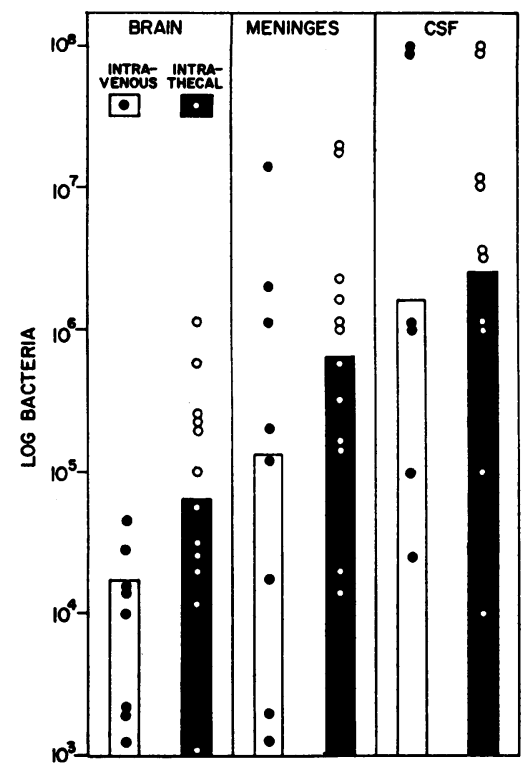

Fig. 6. Bacterial counts of CSF, meninges, and BRAIN IN ANIMALS INFECTED INTRAVENOUSLY AND INTRATHECALLY. In both instances bacterial counts in CSF exceeded those in the meninges, which in turn were greater than those in the brain.

veloped a primary cerebritis with secondary involvement of the coverings of the central nervous system. Therefore, animals were sacrificed at intervals during the infection, the meninges and brain removed aseptically, and simultaneous quantitative bacterial counts of CSF, meninges, and brain were performed. The results are summarized in Figure 6, which indicates that in every animal the number of organisms in the CSF exceeded the number in the meninges, which in turn was always greater than that found in cerebral tissue. This provided evidence that bacteria entered the subarachnoid space directly from the blood and that cerebral infection occurred only secondarily. As might be expected, dogs infected by the intrathecal route also had more bacteria in the CSF and meninges than in the brain.

Pathological changes. Autopsies were performed on nine animals infected during bacteremia. The morphological findings were indistinguishable from those of animals infected intrathecally and have been presented in detail elsewhere (1). The infection involved the entire neuraxis and the exudate was no greater in the cervical cord and the medulla, the areas closest to the needle puncture. Careful microscopic study of the meninges did not reveal the site of entry of the bacteria.
Clinical picture. Except for the slightly more indolent course in intravenously infected dogs, the clinical findings were identical with those observed in animals infected by direct instillation of bacteria into the central nervous system.

Factors influencing development of meningitis during bacteremia. In order to determine the mechanism by which microorganisms entered the subarachnoid space, experiments were performed in which dogs were subjected to maneuvers known to alter the CSF pressure or to affect the permeability of the blood-CSF barrier. These may be summarized as follows.

1) Six dogs were given $250 \mathrm{ml}$ of 6 per cent dextran intravenously over the course of 1 hour. In control animals this had produced a fall in CSF pressure of approximately 50 per cent. After completion of the infusion, $10^{9}$ bacteria were given intravenously. Meningitis did not ensue.

2) Five animals were injected with $100 \mathrm{ml} 5$ per cent dextrose intravenously during a 5-minute period. This procedure resulted in a fall in CSF pressure of 40 to 70 per cent. Then $10^{\circ}$ pneumococci were administered. None of the animals became infected.

3) A blood pressure cuff was placed around the necks of anesthetized dogs and inflated to a pressure of $30 \mathrm{~mm} \mathrm{Hg}$. This regularly resulted in doubling the animals' CSF pressure. The animals were permitted to recover, and 3 days later were given $10^{9}$ pneumococci intravenously with the cuff inflated. Meningitis did not result.

4) Six animals were given $2.0 \mathrm{ml}$ of heat-killed typhoid vaccine containing $10^{10}$ bacteria. Two hours later, when the animals were severely ill with fever, diarrhea, and lethargy, $10^{9}$ pneumococci were injected intravenously. The central nervous system remained free of infection. Previous experiments had demonstrated a marked increase in the permeability of the blood-CSF barrier as measured by penetration of sodium fluorescein from blood to CSF in animals given typhoid vaccine (9). Eckman, King and Brunson have also demonstrated an increase in the permeability of the blood-CSF barrier after administration of bacterial endotoxins (10).

5) Aseptic meningitis was produced in four dogs by administration of $6.0 \mathrm{ml} 0.85$ per cent $\mathrm{NaCl}$ into the cisterna magna (11). Four hours later these animals had an average of 5,800 polymorphonuclear leukocytes per $\mathrm{mm}^{3} \mathrm{CSF}$. Pre- 
vious studies had demonstrated that exudation of this magnitude was always associated with an increase in permeability of the blood-CSF barrier, as measured by transfer of sodium fluorescein from blood to CSF (9). Five hours after induction of aseptic meningitis ( 1 hour after the preceding cisternal puncture), $7.6 \times 10^{7}$ pneumococci were given intravenously. Cisternal punctures 4 and 24 hours later did not reveal evidence of meningeal infection.

The preceding five experiments, in three of which CSF pressure was drastically altered, and two of which were associated with marked increments in the blood-CSF barrier, failed to facilitate development of meningeal infection during massive pneumococcal bacteremia. In none of these was cisternal puncture performed within 30 minutes of intravenous inoculation of bacteria.

To determine whether these alterations might increase the rate of infection in animals subjected to lumbar puncture during bacteremia, nine animals were given typhoid vaccine 2 hours before cisternal puncture and bacteremia, eight were infused with dextran before cisternal puncture and injection of bacteria, and two were given $100 \mathrm{ml}$ 50 per cent dextrose. Forty-three animals, subjected to cisternal puncture during bacteremia, but not given any adjuvants, served as controls. The results, which are summarized in Table II, show that these maneuvers did not alter the rate of infection.

Effect of initial, final, and change in CSF pressure on development of infection (Figure 7). Although approximately $2.0 \mathrm{ml}$ of CSF was removed during each cisternal puncture, there was marked individual variation in the animals' CSF pressure at the beginning of cisternal puncture, after removal of fluid, and in the magnitude of the change in CSF pressure effected by release of CSF. There was no correlation between these indices

TABLE II

Effect on development of meningitis of procedures known to alter CSF pressure and permeability of blood-CSF barrier plus cisternal puncture (C.P.) during pneumococcal bacteremia

\begin{tabular}{|c|c|c|c|}
\hline & $\begin{array}{l}\text { No. } \\
\text { animals }\end{array}$ & $\begin{array}{c}\text { No. } \\
\text { infected }\end{array}$ & $\begin{array}{l}\text { Per cent } \\
\text { infected }\end{array}$ \\
\hline No adjuvants + C.P. & 43 & 22 & 51 \\
\hline $\left.\begin{array}{l}\text { Typhoid vaccine } \\
6 \% \text { dextran } \\
50 \% \text { dextrose }\end{array}\right\}+$ C.P. & 19 & 9 & 47 \\
\hline
\end{tabular}

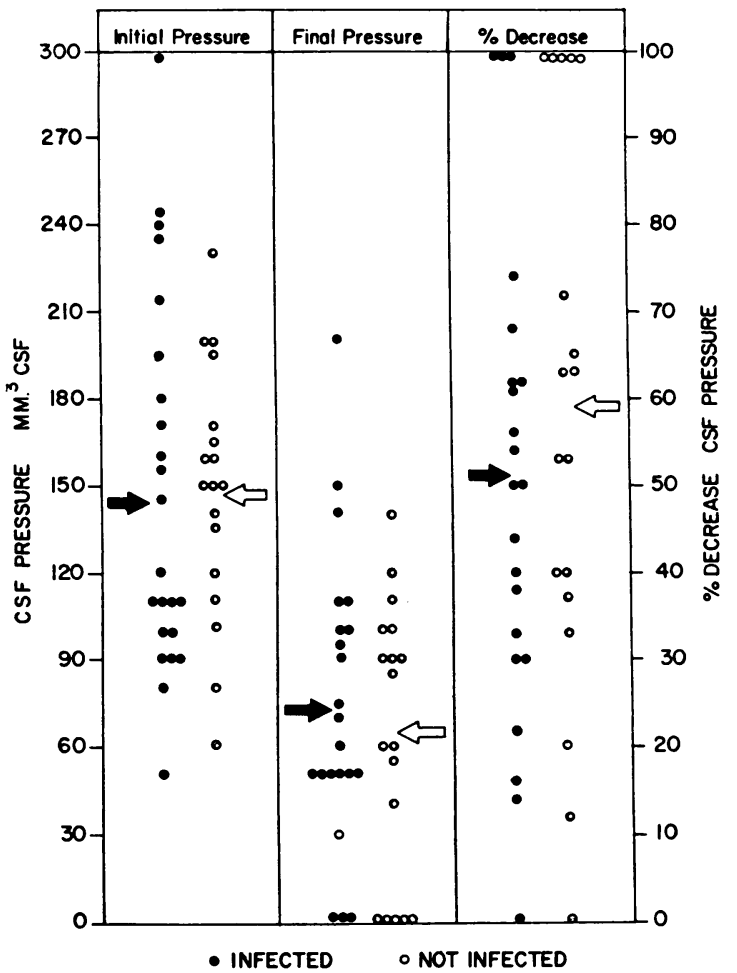

Fig. 7. Initial, Final, AND MAgNitude of ChANGe in CSF PRESSURES IN ANIMALS SUBJECTED to CISTERNAL PUNCTURE DURING PNEUMOCOCCAL BACTEREMIA. There was no difference between these parameters in infected animals and in those who did not develop meningitis.

in CSF pressure and development of infection (Figure 7).

If the fall in CSF pressure occasioned by the release of CSF is a prerequisite for infection, then replacement of fluid immediately after its removal should militate against development of meningeal infection during bacteremia. After administration of $10^{8}$ pneumococci intravenously, six dogs were subjected to cisternal puncture, and $2.0 \mathrm{ml}$ of fluid removed. The mean decrease in CSF pressure in these animals was $50 \mathrm{~mm}$ CSF. Immediately thereafter the CSF was replaced with return of CSF pressure to within 10 per cent of the baseline level. Five of the six animals developed meningitis despite replacement of fluid.

\section{DISCUSSION}

These studies demonstrate that microtrauma to the meninges in the form of cisternal puncture during pneumococcal bacteremia results in meningitis provided sufficient numbers of microorganisms are in the blood stream at the time of cisternal puncture. These results are in agree- 
ment with those obtained by Weed and associates with $A$. aerogenes in cats $(6,8)$. However, the data permit only one explanation for the mechanism of entry of microorganisms, namely, that the bacteria entered the subarachnoid space through the small opening made at the time of cisternal puncture. The finding that development of infection was directly related to the number of organisms in the blood stream at the time of needle puncture and the fact that infection only occurred within 30 minutes of cisternal puncture - presumably because the hole is sealed off after that interval-favor this idea.

In contrast, Weed and his co-workers postulated that direct trauma was not the mechanism of entry of bacteria, but that an acute alteration in the volume of fluid in the subarachnoid space and in the choroid plexus consequent to release of CSF facilitated infection. They marshalled the following evidence to support this hypothesis $(6,8)$ : 1) inability to demonstrate the site of entry, despite careful microscopic studies (12);2) failure to localize cells and bacteria at the base of the brain, close to the site of the needle track; 3) removal and replacement of CSF 30 minutes before intravenous administration of bacteria prevented development of meningitis, but the same maneuver 30 minutes after bacteremia did not interfere with the occurrence of the infection; and 4) infusion of hypertonic saline solutions, jugular compression, and cardiac standstill during bacteremia resulted in meningitis without cisternal puncture.

Our results confirm the failure to find the point of entry, but this is not surprising since it seems likely that the hole made by the needle was sealed off rapidly, making it difficult, if not impossible, to find. The same explanation can be invoked to account for the differences observed when CSF was replaced before and after bacteremia. In the former instance the defect in the meninges had closed before bacteria were in the blood stream, while replacement of fluid failed to prevent infection after microorganisms had been injected intravenously. It is also not surprising that meningitis ensued when bacteria were injected after administration of hypertonic saline, since it has been demonstrated that infusion of hyperosmolar solutions of electrolytes into cats may cause diffuse hemorrhagic encephalopathy (13).

In our studies, no procedure other than direct trauma to the meninges (i.e., cisternal puncture) was followed by infection. The divergence between the observations of Weed and colleagues and the present findings may be due to the fact that $A$. aerogenes is more virulent for the meninges than $D$. pneumoniae. Although $A$. aerogenes undoubtedly elaborated a strong endotoxin, it is unlikely that meningeal virulence is dependent upon this toxin per se, because administration of large doses of typhoid vaccine prior to pneumococcal bacteremia did not enhance passage of bacteria from blood to CSF. It is also possible that the large encapsulated pneumococcus was unable to penetrate the blood-CSF barrier through the submicroscopic defects resulting from infusions of hypertonic solutions, while these produced sufficient trauma to permit entry of the smaller gram-negative bacillus. Finally, the leptomeninges of the cat may be more delicate, and thus more permeable, than those of the dog.

These experimental results are not inconsistent, however, with the clinical axiom that pneumococcal meningitis usually occurs in a setting where a small communication between the subarachnoid space and the external environment may be presumed to exist and where the meninges are not in their pristine state as in sinusitis, mastoiditis, otitis, head trauma, and so forth. It is certainly not difficult to visualize that microtrauma does occur even in patients with no discernible portal of entry. It should be pointed out, however, that these studies are only applicable to one bacterial genus, the pneumococcus, and to one animal species, the dog, and that they will have to be extended to other species and microorganisms.

Failure to find a relationship between CSF pressure and development of infection in the present study may be due to failure of the methods employed for measuring CSF pressure to detect subtle changes in CSF dynamics. Perhaps implantation of a catheter in the subarachnoid space with repeated measurements of pressure may overcome this problem. However, the catheter would act as a foreign body and would be comparable to needle puncture in facilitating entry of bacteria from the blood stream.

Also of clinical significance is the possible danger of diagnostic lumbar puncture during bacteremia. Wegeforth and Latham (14) performed lumbar punctures in 93 patients with suspected meningitis; in 55 of these cerebrospinal fluid was 
normal. Six patients had meningococcemia or pneumococcal bacteremia at the time of lumbar puncture and 5 of the 6 subsequently developed meningitis. On the basis of these findings the authors condemn diagnostic lumbar puncture as a distinct hazard in patients with bacteremia. Remsen (15) shared this viewpoint and added a case of streptococcal meningitis occurring under similar circumstances. It seems likely that these few cases are not representative of the over-all clinical experience, and that meningitis following lumbar puncture is relatively rare. Pray (16) did not find an increased incidence of meningitis in children with pneumococcal sepsis who were subjected to lumbar puncture. If the experimental results of the present studies are applied to man, the relative rarity of meningitis complicating bacteremia becomes apparent. The large majority of patients with pneumococcal bacteremia harbor between 2 and 100 organisms per $\mathrm{ml}$ of blood, an inoculum too small to produce infection in experimental animals. The present data do not support the idea that suspected or documented bacteremia constitutes a contraindication to lumbar puncture. Compared with the risk of missing the diagnosis of meningitis by omission of lumbar puncture, the chance of developing meningeal infection after the procedure is small.

\section{SUMMARY}

Type III pneumococcal meningitis was produced in dogs by performance of cisternal puncture within 2 minutes after intravenous administration of pneumococci. Development of infection was related to the size of the inoculum and, even more critically, to the number of organisms in the blood stream at the time CSF was released. The infection was characterized by progressive increase in cells and bacteria in the CSF and was indistinguishable clinically and pathologically from meningitis produced by direct instillation of pneumococci into the subarachnoid space. Procedures which altered CSF pressure, such as infusion of 6 per cent dextran, 50 per cent dextrose, and jugular compression, and procedures which enhanced the permeability of the blood-CSF barrier (i.e., administration of typhoid vaccine and induction of aseptic meningitis) did not result in meningitis during bacteremia, and direct trauma to the meninges in the form of cis- ternal puncture was the only stimulus which permitted entry of bacteria into the subarachnoid space.

\section{REFERENCES}

1. Petersdorf, R. G., and Luttrell, C. N. Studies on the pathogenesis of meningitis. I. Intrathecal infection. J. clin. Invest. 1962, 41, 311.

2. Bull, C. G. Immunity factors in pneumococcus infection in the dog. J. exp. Med. 1916, 24, 7.

3. Amoss, H. L., and Eberson, F. Experiments on the mode of infection in epidemic meningitis. J. exp. Med. 1919, 29, 605.

4. Austrian, C. R. Experimental meningococcus meningitis. Bull. Johns Hopk. Hosp. 1918, 29, 183.

5. Idzumi, G. Experimental pneumococcus meningitis in rabbits and dogs. J. infect. Dis. 1920, 26, 373.

6. Weed, L. H., Wegeforth, P., Ayer, J. B., and Felton, L. D. A study of experimental meningitis. IV. The influence of certain experimental procedures upon the production of meningitis by intravenous inoculation. Monogr. Rockefeller Inst. med. Res. 1920, 12, 57.

7. Wollstein, M. Influenzal meningitis and its experimental production. Amer. J. Dis. Child. 1911, 1, 42.

8. Weed, L. H., Wegeforth, P., Ayer, J. B., and Felton, L. D. The production of meningitis by release of cerebrospinal fluid during an experimental septicemia: Preliminary note. J. Amer. med. Ass. 1919, 72, 190.

9. Swarner, D. R., and Petersdorf, R. G. Unpublished observations.

10. Eckman, P. L., King, W. M., and Brunson, J. G. Studies on the blood brain barrier. I. Effects produced by a single injection of gram-negative endotoxin on the permeability of the cerebral vessels. Amer. J. Path. 1958, 34, 631.

11. Petersdorf, R. G., and Harter, D. H. The fall in cerebrospinal fluid sugar in meningitis. Some experimental observations. Arch. Neurol. Psychiat. (Chicago) 1961, 4, 21.

12. Ayer, J. B. A study of experimental meningitis. V. Experimental acute hematogenous meningitis. A pathological study. Monogr. Rockefeller Inst. med. Res. 1920, 12, 113.

13. Luttrell, C. N., Finberg, L., and Drawdy, L. P. Hemorrhagic encephalopathy introduced by hypernatremia. II. Experimental observations on hyperosmolarity in cats. A.M.A. Arch. Neurol. 1959, 1, 153.

14. Wegeforth, P., and Latham, J. R. Lumbar puncture as a factor in the causation of meningitis. Amer. J. med. Sci. 1919, 158, 183.

15. Remsen, D. B. The rôle of lumbar puncture in the causation of meningitis. J. Med. 1936, 17, 115.

16. Pray, L. G. Lumbar puncture as a factor in the pathogenesis of meningitis. A.M.A. J. Dis. Child., $1941,62,295$. 